\title{
Propriedades mecânicas do MDP produzido com partículas de madeira de Ilex paraguariensis, Pinus elliottii e Eucalyptus grandis
}

\author{
Mechanical properties of MDP produced with wood particles of llex \\ paraguariensis, Pinus elliottii e Eucalyptus grandis
}

\author{
Joel Telles de Souza ${ }^{1}$, Maiara Talgatti ${ }^{1 *}$, Amanda Grassmann da Silveira ${ }^{1}$, \\ Walmir Marques de Menezes ${ }^{1}$, Clóvis Roberto Haseleinn ${ }^{1}$ e Elio José Santini ${ }^{1}$
}

\begin{abstract}
Resumo
O presente estudo tem como objetivo avaliar a viabilidade da utilização de resíduos madeireiros da industrialização da erva-mate (llex paraguariensis a. st. hil.), para a manufatura de painéis MDP, em combinação com diferentes proporções de madeira de Eucalyptus grandis e Pinus elliottii, coladas com ureia-formaldeído. Para realização deste trabalho, foram utilizados resíduos madeireiros de erva-mate e partículas de madeira de pinus e eucalipto. Os ensaios mecânicos de módulo de elasticidade (MOE) e módulo de ruptura (MOR) de flexão estática, arrancamento de parafusos (AP), tração perpendicular à superfície do painel (TP) e resistência superficial (RS). Para este trabalho foi utilizado o modelo experimental conhecido como planejamento em rede centroide simplex, onde é possível predizer o comportamento de uma propriedade a partir dos resultados obtidos com a utilização de cada componente individual. A modelagem se manifestou da seguinte maneira: MOR de flexão estática, AP e TP são determinados pelo modelo linear, enquanto, MOE de flexão estática e RS são determinados por modelo quadrático. De maneira geral, o acréscimo de partículas de erva-mate incorreu em redução na maioria das propriedades mecânicas do MDP. No entanto, somente o tratamento com $100 \%$ de erva-mate apresentou desempenho inferior ao exigido pela norma brasileira ABNT-Associação Brasileira de Normas Técnicas, na maior parte dos ensaios realizados. A proporção mais indicada para a confecção de painéis é a mistura de $33,33 \%$ de partículas de cada espécie. Sendo assim, conclui-se que, o resíduo madeireiro de erva-mate em combinação com as espécies comumente utilizadas em painéis comerciais, pinus e eucalipto, pode ser utilizado em painéis MDP sem prejudicar a qualidade dos mesmos.
\end{abstract}

Palavras-chave: Resíduos, painéis aglomerados, ureia-formaldeído, modelagem.

\begin{abstract}
The present study aims to evaluate the feasibility of the use of wood residues from the industrialization of yerba mate (Ilex paraguariensis a. St hil.), for the manufacture of MDP panels, in combination with different proportions of wood of Eucalyptus grandis and Pinus elliottii, bonded with urea-formaldehyde. In order to carry out this research, wood residues of yerba mate and pine and eucalyptus wood particles. The mechanical tests of modulus of elasticity (MOE) and modulus of rupture (MOR) of static bending, pulling of bolts (AP), traction perpendicular to the surface of the panel (TP) and Surface resistance (RS). For this study we used the experimental model known as simplex centroid network planning, where it is possible to predict the behavior of a property from the results obtained with the use of each individual component. The modeling was manifested as follows: static bending MOR, AP and TP are determined by the linear model, whereas static bending MOE and RS are determined by quadratic model. In general, the addition of yerba mate particles has reduced most of the mechanical properties of MDP. However, only the treatment with $100 \%$ of yerba mate presented a performance lower than that required by the Brazilian standard ABNT - Brazilian Association of Technical Norms, in most of the tests performed. The most suitable proportion for the preparation of panels is the mixing of $33,33 \%$ of particles of each species. Therefore, it is concluded that the woody residue of yerba mate in combination with the species commonly used in commercial panels, pine and eucalyptus, can be used in MDP panels without harming the quality of the same.
\end{abstract}

Keywords: Waste, particleboards, urea-formaldehyde, modeling.

1. Departamento de Ciências Florestais, Universidade Federal de Santa Maria - UFSM. Santa Maria / RS, Brasil. * Autor Correspondente: maiara.talgatti@hotmail.com 
Talgatti et al. - Propriedades mecânicas do MDP produzido com partículas de

madeira de Ilex paraguariensis, Pinus elliottii e Eucalyptus grandis

\section{INTRODUÇÃO}

Um dos grandes consumidores de madeira no setor florestal, é a indústria de painéis de partículas. Dentre os painéis de madeira reconstituída, o MDP (medium density particleboard) é um dos mais produzidos e consumido no mundo (BIAZUS et al., 2010). No Brasil representa uma produção anual de 3 milhões de metros cúbicos (ABIPA, 2015), estando entre os 10 maiores produtores em escala mundial. A importância mercadológica do MDP se concentra no abastecimento de produtoras de móveis para uso residencial e comercial, onde este produto se mostra como uma das principais matéria-prima. Ainda, de acordo com o relatório da Indústria Brasileira de Árvores- IBÁ (2017), no ano de 2016, a produção brasileira de painéis de madeira reconstituída registrou redução de 2,4\% em relação ao anterior e encerrou o ano com 7,3 milhões de $\mathrm{m}^{3}$ de produzidos. As produções de MDF diminuíram 8,8\%, enquanto a produção de MDP aumentou 8,9\%. Com o crescimento da produção industrial de painéis de madeira, aumenta, também, a demanda por matéria-prima, o que se torna necessário não apenas o aumento de áreas de plantios com espécies atualmente utilizadas, mas a busca de novas opções. Uma das alternativas pode ser os resíduos madeireiros, como da industrialização da erva-mate, principal proposta deste trabalho.

Segundo Muruganandam, Ranjitha e Harshavardhan (2016), o painel de partículas pode ser fabricado a partir de uma variedade de materiais sob a forma de partículas, que são unidas com adesivos sintéticos ou outros ligantes apropriados reforçados com calor e pressão e podem ser utilizados para diversas finalidades dependo do material utilizado. Corroborando Mendes et al. (2009), entre os resíduos com potencial para produção de aglomerados, destacam-se o sabugo de milho, as cascas de arroz, café, amendoim, mamona, coco, o pseudocaule de bananeira, o caule da mandioca e o bagaço de cana.

De acordo com Silva et al. (2015), a confecção de painéis com os mais diversos resíduos agrícolas mostra-se como uma alternativa ecologicamente favorável para o desenvolvimento tecnológico do setor de painéis, com materiais competitivos e de propriedades físicas e mecânicas equiparadas aos produtos tradicionais encontrados no mercado. Além disso, a utilização desses resíduos permite agregar valor e favorece a melhoria social de pequenos produtores agrícolas, gerando renda a partir de materiais antes descartados. Os autores Talgatti et al. (2017), destacam que a diversificação das bases para a fabricação de compósitos reconstruídos, por meio de novos produtos, poderá beneficiar o setor florestal, ao passo que a fabricação industrial poderá aumentar, sem que haja necessariamente a implantação de novos maciços florestais.

Ainda, pesquisadores como, Baldin et al. (2018), Carvalho et al. (2015), Iwakiri et al. (2017), Sanches et al. (2016) e Silva et al. (2015) vêm buscando alternativas para substituir ou complementar a madeira na manufatura destes produtos. A mescla de partículas de madeira com outros materiais lignocelulósicos para produção de painéis objetiva a redução nos custos de obtenção da matéria-prima e pode ser empregada em escala industrial, desde que não sejam alteradas negativamente as propriedades físico-mecânicas destes produtos ou então, que seja estabelecida uma quantidade máxima de adição destes materiais sem prejuízos de qualidade.

As propriedades mecânicas de MDP variam conforme a sua composição, espécie de madeira utilizada, diferentes proporções de cada espécie em um mesmo painel, formação das diferentes camadas, quantidade de adesivo e critérios de produção, desde a formação das partículas até a prensagem e resfriamento dos painéis. Consoante ao exposto, encontra-se a variação dos resultados das propriedades tecnológicas observadas na literatura pelos mais diversos trabalhos realizados com MDP, utilizando espécies florestais madeireiras puras ou em mistura com materiais lignocelulósicos como matéria-prima fabril de painéis. Desta maneira, esta pesquisa apresentou a finalidade de verificar as propriedades mecânicas, em painéis com três camadas compostas por resíduos madeireiros da erva-mate (Ilex paraguariensis), Eucalyptus grandis e Pinus elliottii.

\section{MATERIAL E MÉTODOS}

Para realização deste trabalho, foram utilizadas partículas de madeira de Ilex paraguariensis A. St. Hil., Eucalyptus grandis W. Hill ex Maiden e Pinus elliottii Engelm e adesivo à base de ureia-formaldeído industrialmente produzido com um teor de sólidos de aproximadamente 65\%. 
O resíduo madeireiro de erva-mate foi cedido pela empresa Ervateira Barão de Cotegipe Ltda., da cidade de Barão de Cotegipe- RS. O resíduo era constituído de talos e palitos de diversos tamanhos, isento de casca. Este material foi processado em moinho de martelos equipado de peneira com orifícios de 8,0 mm de diâmetro, para as partículas de camada interna (Figura 1c); e peneira de 1,0 mm de diâmetro, para as partículas de camada externa (Figura 1b). Por fim, o material foi submetido à peneiragem com abertura de malha de 0,150 mm, para descarte das partículas mais finas (Figura 1a). As partículas utilizadas foram as que não passaram pela malha da peneira.

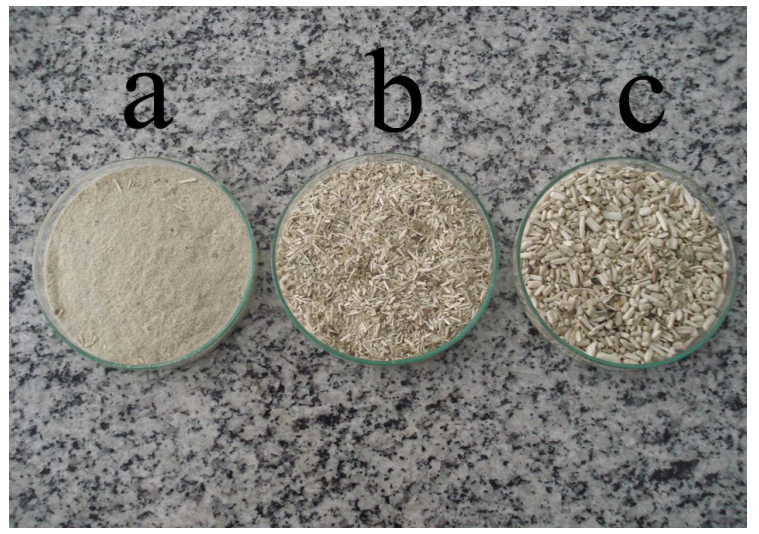

Figura 1 - Resíduo madeireiro de erva-mate após o processamento e peneiragem. Descarte das partículas mais finas - pó (a); partículas da camada externa (b) e partículas da camada interna (c).

Figure 1 - Yarrow wood residue after processing and sieving. Discarding the finer particles - powder (a); particles of the outer layer (b) and particles of the inner layer (c).

Já as árvores de Eucalyptus grandis e Pinus elliottii foram obtidas em povoamentos florestais homogêneos de aproximadamente 15 anos, localizados sob as coordenadas $29^{\circ} 41^{\prime} 29^{\prime \prime}$ S, 53 $3^{\circ} 48^{\prime} 3^{\prime \prime}$ O. Foram selecionadas três árvores de cada espécie, de fuste reto e boas condições fitossanitárias e retirada as duas primeiras toras com 2,70 m cada. Procedeu-se ao desdobro das toras com a retirada de tábuas de $5 \mathrm{~cm}$ de espessura, transformadas em blocos. Já a obtenção das lascas foi realizada em flaker, com corte ajustado para $0,55 \mathrm{~mm}$ de espessura. Este material foi submetido à secagem ao ar por 15 dias e reduzido a partículas em moinho de martelos, equipado de peneira com orifícios de 8,0 mm de diâmetro, para as partículas de camada interna (Figura 2c); e peneira de 1,0 mm de diâmetro, para as partículas de camada externa (Figura 2b). Por fim, o material foi peneirado com abertura de malha de 0,150 mm, para descarte das partículas mais finas (Figura 2a).

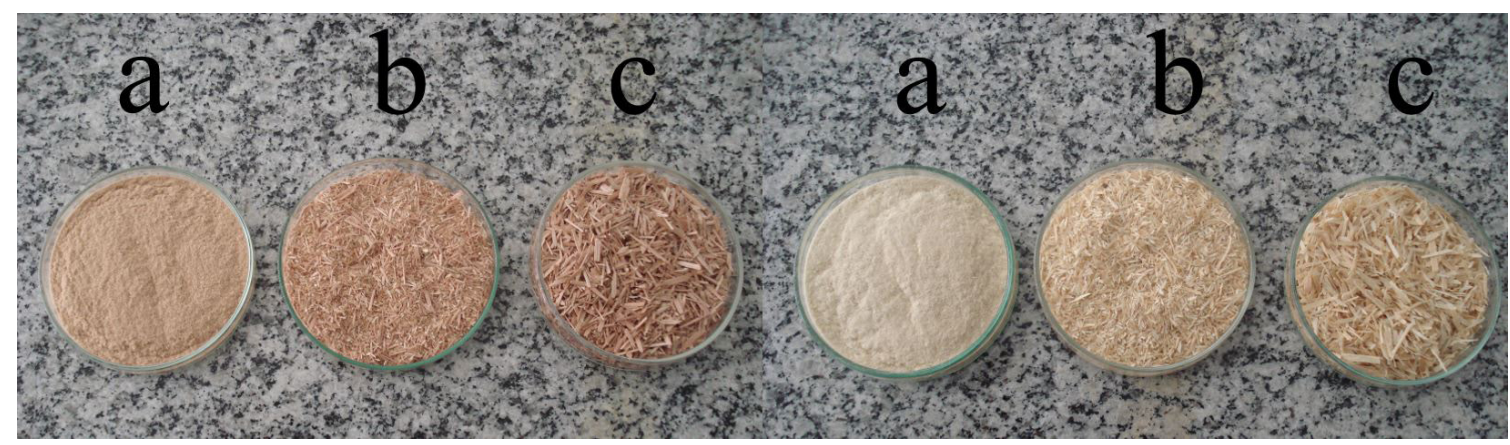

Figura 2 - Partículas de Eucalyptus grandis (à esquerda em coloração rosada) e Pinus elliottii (à direita em coloração amarelada), após o processamento e peneiragem. Descarte das partículas mais finas - pó (a); partículas da camada externa (b) e partículas da camada interna (c).

Figure 2 - Eucalyptus grandis (left in pink) and Pinus elliottii (right in yellow), after processing and sieving. Discarding the finer particles - powder (a); particles of the outer layer (b) and particles of the inner layer (c).

Fonte: $\quad$ Adaptado de Borsato et al. 2010.

Após a preparação das partículas, as mesmas foram condicionadas em estufa a $60^{\circ} \mathrm{C}$ durante pelo menos 24 horas, até alcançar a umidade de aproximadamente 3\%. Como se trata de painel MDP em camadas, a composição do mesmo foi pré-estabelecida da seguinte maneira: 
Talgatti et al. - Propriedades mecânicas do MDP produzido com partículas de

madeira de Ilex paraguariensis, Pinus elliottii e Eucalyptus grandis

- Camada externa (CE): 86\% de partículas, 13\% de adesivo e 1\% de parafina;

- Camada interna (CI): 91\% de partículas, $8 \%$ de adesivo e $1 \%$ de parafina.

Para ambas as camadas, trabalhou-se com uma massa específica de 0,65 g.cm-3 com umidade de equilíbrio de $8 \%$. As partículas de cada camada foram levadas a um tambor rotativo e misturadas nas diferentes proporções. Juntamente com o adesivo e a parafina, foi aplicada a água necessária para ajustar a umidade do colchão a, aproximadamente, $12 \%$ para CE e $8 \%$, para CI. Gerada a mistura que dava origem ao colchão, a mesma foi pesada e levada a um molde de madeira de $50 \times 50 \times 20 \mathrm{~cm}$, onde foi realizada uma pré-prensagem manual a temperatura ambiente por 2 minutos. Nesta etapa procedeu-se da seguinte maneira para a formação do colchão:

- $\quad \mathrm{CE}=44 \%$ : - Inferior: $21 \%$

- Superior: $23 \%$

- $\quad \mathrm{CI}=56 \%$

Posteriormente, o colchão foi submetido a prensagem definitiva, realizada em prensa hidráulica com capacidade para aplicação de carga de 100 toneladas e pratos com dimensões de 60 x $60 \mathrm{~cm}$. Foi aplicada uma pressão especifica de $30 \mathrm{kgf.cm}^{-2}$ e a temperatura dos pratos ajustada para $180^{\circ} \mathrm{C}$.

Os ensaios mecânicos, assim como o tamanho dos corpos de prova (CPs) para cada teste, foram desenvolvidos conforme a norma ABNT NBR 14810-2 (ABNT, 2013) da Associação Brasileira de Normas Técnicas (Tabela 1). Todos estes testes foram realizados no laboratório da empresa Duratex S/A, planta de Taquari - RS.

Tabela 1 - Dimensões dos corpos de prova que foram utilizados na determinação das propriedades mecânicas, velocidade dos ensaios e norma utilizada.

Table 1 - Dimensions of the specimens that were used in the determination of the mechanical properties, speed of the tests and standard used.

\begin{tabular}{lcc}
\hline \multicolumn{1}{c}{ Propriedades mecânicas } & $\begin{array}{c}\text { Dimensões dos corpos de } \\
\text { prova (cm) }\end{array}$ & $\begin{array}{c}\text { Velocidade do } \\
\text { ensaio (mm/min) }\end{array}$ \\
\hline Flexão estática & $25 \times 5 \times 1,5$ & 7,7 \\
Arrancamento de parafuso de topo e face & - & 15 \\
Tração perpendicular & $5 \times 5 \times 1,5$ & 4 \\
Resistência superficial & $5 \times 5 \times 1,5$ & 5 \\
\hline
\end{tabular}

Utilizaram-se os mesmos corpos de prova do teste de flexão estática para o ensaio de arracamento de parfuso. Para isso, depois de rompidos, foram retirados os dois extremos de cada CP, que não foram afetados pela ruptura e colados um sob o outro. Esta nova amostra foi, então, perfurada com auxílio de uma broca de 2,8 $\mathrm{mm}$ e com profundidade de $17 \mathrm{~mm}$, centrado na superfície e topo do corpo de prova. Foi introduzido o parafuso específico para o teste, antes na face, onde se obteve os dados de arrancamento de parafusos na face (AP Face), após no topo, onde se obteve os dados de arrancamento de parafusos no topo (AP Topo).

Foram utilizados corpos de prova com dimensões descritas na Tabela 1 para aferição da tração perpendicular à superfície do painel (TP), cuja as faces foram coladas em blocos de metal com adesivo termofusível (cola hot-melt).

Para o ensaio de resistência superficial (RS), no centro geométrico de cada corpo de prova, foi confeccionado uma ranhura anelar de 35,7 mm de diâmetro e profundidade de 0,3 a 1,0 mm. Após com o uso de adesivo hot-melt, foi colado sobre a superfície ranhurada, um dispositivo metálico de diâmetro semelhante à ranhura realizada. Posteriormente, a cura total do adesivo, foi acoplado o conjunto nos acessórios da máquina universal de ensaios e realizado o teste de resistência superficial.

\section{Delineamento experimental}

Objetivando avaliar o efeito das partículas madeireiras de erva-mate na manufatura de painéis MDP, foram combinadas sete diferentes proporções de partículas de Ilex paraguariensis, com Eucalyptus grandis e Pinus elliottii, coladas com ureia-formaldeído. Portanto, foram avaliados sete tratamentos de painéis MDP (Tabela 1), com densidade nominal de $0,65 \mathrm{~g}_{\mathrm{cm}} \mathrm{cm}^{-3}$.

Para o delineamento centróide simplex, metodologia baseada no estudo de HILLIG, et al. (2006), apenas os tratamentos com quantidades de 0; 33,33; 50 e 100\% de erva-mate, pinus e eucalipto 


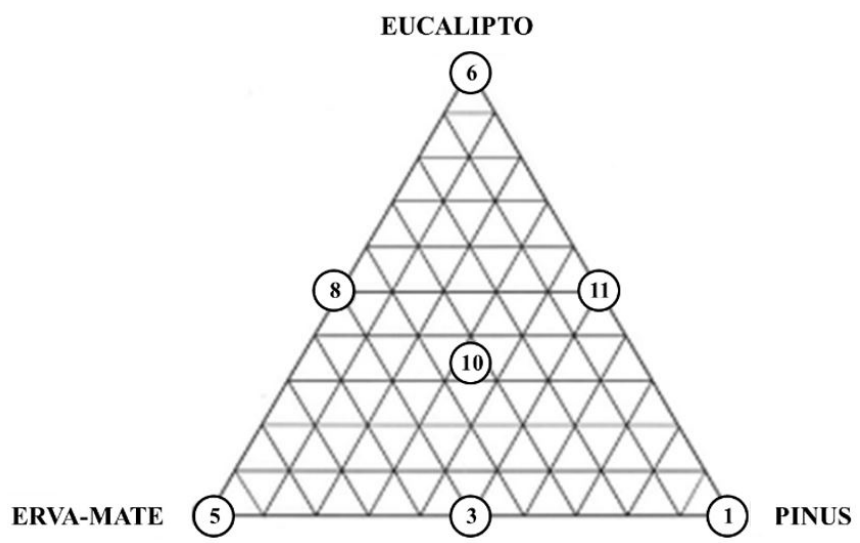

Figura 3 - Delineamento experimental centróide simplex para três componentes.

Figure 3 - Simplex centroid experimental design for three components.

foram utilizados, sendo, portanto, os tratamentos T1, T3, T5, T6, T8, T10 e T11 (Figura 3). Para cada mistura, foram realizadas quatro repetições. Destaca-se que a mistura 1 é 100\% pinus; mistura 5, $100 \%$ erva-mate; mistura 6, 100\% eucalipto; mistura 3, 8 e 11, proporções iguais de duas espécies e mistura 10, proporções iguais das três espécies.

\section{Análise estatística dos dados}

O modelo experimental utilizado é conhecido como planejamento em rede centróide simplex. Nesse delineamento, o modelo mais simples para uma mistura é o modelo linear ou aditivo, que procura explicar o comportamento de uma propriedade apenas com os resultados obtidos com a utilização de cada componente individualmente, isto é, prever o comportamento de qualquer mistura sem realizar nenhuma mistura. Numa mistura de três componentes, pode-se ter, além deste, modelos quadráticos que consideram os efeitos das interações de dois componentes e modelos cúbicos que consideram os efeitos das interações dos três componentes.

Sendo assim, os modelos utilizados foram expressos nas Equações derivadas 1, 2 e 3, respectivamente.

$$
\begin{gathered}
\mathrm{Yi}=\mathrm{b} 1 . \mathrm{X} 1+\mathrm{b} 2 . \mathrm{X} 2+\mathrm{b} 3 \cdot \mathrm{X} 3 \\
\mathrm{Yi}=\mathrm{b} 1 . \mathrm{X} 1+\mathrm{b} 2 . \mathrm{X} 2+\mathrm{b} 3 . \mathrm{X} 3+\mathrm{b} 12 . \mathrm{X} 1 . \mathrm{X} 2+\mathrm{b} 13 . \mathrm{X} 1 . \mathrm{X} 3+\mathrm{b} 23 . \mathrm{X} 2 . \mathrm{X} 3 \\
\mathrm{Yi}=\mathrm{b} 1 . \mathrm{X} 1+\mathrm{b} 2 . \mathrm{X} 2+\mathrm{b} 3 \cdot \mathrm{X} 3+\mathrm{b} 12 . \mathrm{X} 1 . \mathrm{X} 2+\mathrm{b} 13 . \mathrm{X} 1 . \mathrm{X} 3+\mathrm{b} 23 . \mathrm{X} 2 . \mathrm{X} 3+\mathrm{b} 123 . \mathrm{X} 1 . \mathrm{X} 2 . \mathrm{X} 3
\end{gathered}
$$

Em que: $Y i=$ Variável resposta; $b i=$ Coeficientes; $X i=$ Proporção de cada espécie na mistura.

Para a determinação dos coeficientes, foram utilizadas as sete misturas mínimas necessárias para o modelo cúbico, conforme Tabela 1 e Figura 3. Na avaliação dos modelos, foram testados modelos cúbicos para todas as propriedades analisadas, realizada análise da variância, e descartados os coeficientes não-significativos pelo teste " $\mathrm{t}$ ". A partir deste ajuste, foram utilizadas as misturas mínimas necessárias para estimar o modelo, cúbico, quadrático ou linear.

\section{RESULTADOS E DISCUSSÃO}

A Tabela 2 apresenta os resultados médios de MOE e MOR dos painéis confeccionados com a mistura das diferentes espécies, erva-mate, pinus e eucalipto.

É possível observar que os valores de MOE e MOR tiveram variação de 1181,45 a 2435,49 MPa e 4,84 a 14,50 MPa, respectivamente, sendo o menor valor para o tratamento 5 com $100 \%$ de erva-mate, para ambas as propriedades mecânicas. Sendo assim, os painéis fabricados com partículas madeireiras de erva-mate em grande proporção tiveram tendência a possuir valores inferiores para MOE e MOR. Estes resultados podem ser atribuídos a geometria das partículas de erva-mate, que se encontram mais cilíndricas e arredondadas do que as comerciais, este mesmo resultado foi observado por Torrell et al. (2013), avaliando a viabilidade do uso de partículas de serragem em painéis aglomerados de pinus. 
Talgatti et al. - Propriedades mecânicas do MDP produzido com partículas de

madeira de Ilex paraguariensis, Pinus elliottii e Eucalyptus grandis

Carvalho et al. (2015), estudando diferentes proporções de erva-mate e pinus, também encontraram valores inferiores de MOE e MOR, 1180,10 e 13,60 MPa respectivamente, para painéis com 100\% erva-mate. Os referidos autores observaram valores máximos de 1611,70 para MOE e 19,60 MPa para MOR, em painéis com 100\% pinus. Conforme pode ser visto na Tabela 2, o presente estudo encontrou valor superior em painéis puros de pinus, além disso, painéis com porcentagens menores de erva-mate tiveram valores superiores tanto para MOE quanto para MOR, do que o valor máximo encontrado por estes autores.

Tabela 2- Tratamentos utilizados no experimento.

Table 2- Treatments used in the experiment.

\begin{tabular}{cccc}
\hline \multirow{2}{*}{ Tratamentos } & \multicolumn{1}{c}{ Partículas (\%) } & Eucalipto \\
\cline { 2 - 4 } & Erva-mate & Pinus & 0 \\
T1 & 0 & 100 & 0 \\
T3 & 50 & 50 & 0 \\
T5 & 100 & 0 & 100 \\
T6 & 0 & 0 & 50 \\
T8 & 50 & 0 & 33,33 \\
T10 & 33,33 & 33,33 & 50 \\
T11 & 0 & 50 & \\
\hline
\end{tabular}

Os valores encontrados no ensaio de flexão estática, para grande maioria dos tratamentos avaliados, se apresentaram superiores aos encontrados na literatura para outras espécies alternativas. Guimarães Júnior et al. (2013), estudando painéis aglomerados puros de Acacia mangium, obtiveram os valores de 1803,8 e 11,1 MPa para MOE e MOR, respectivamente. Iwakiri et al. (2012a), em estudo com painéis aglomerados confeccionados a partir de resíduos de serrarias de nove espécies de madeiras tropicais da Amazônia verificaram valores médios de 2701,77 MPa para MOE e 19,57 MPa para MOR. Os autores supracitados utilizaram densidade nominal dos painéis maiores (700 a $800 \mathrm{~kg} \cdot \mathrm{m}^{-3}$ ) que o presente estudo $\left(650 \mathrm{~kg} \cdot \mathrm{m}^{-3}\right)$, apresentando valores inferiores a maioria dos tratamentos avaliados, destacando que dentre muitos materiais alternativos a confecção de painéis a erva-mate apresenta resultados dentro dos citados na literatura.

A norma brasileira ABNT NBR 14810-2 (ABNT, 2013), normatiza o MOE e MOR com valores mínimos de $1600 \mathrm{MPa}$ e $11 \mathrm{MPa}$, respectivamente. A maioria dos tratamentos ficaram dentro do exigido ou, se assemelharam com a norma, somente o tratamento 5, com 100\% erva-mate, não atingiu o exigido em pelo menos uma das características mecânicas de flexão estática. Sendo assim, embora a inserção de partículas de erva-mate diminua as propriedades de MOE e MOR, se utilizada a referida espécie em combinação com eucalipto e principalmente pinus, os painéis apresentaram propriedades mecânicas que atingiram valores superiores ao exigido pela norma brasileira supracitada. Tal fato pode estar relacionado a características do material que se trata de um resíduo da indústria ervateira, o qual deriva em sua grande maioria de galhos finos, menos resistentes. Esta mistura de espécies é melhor demonstrada por meio da modelagem de misturas.

Na Tabela 3, são apresentados os modelos simplificados, nos quais foram descartados os coeficientes não-significativos pelo teste " $\mathrm{t}$ " de student, a $5 \%$ de probabilidade de erro, para as propriedades de MOE e MOR.

Tabela 3 - Valores médios de módulo de elasticidade e módulo de ruptura, nos tratamentos avaliados.

Table 3 - Mean values of modulus of elasticity and modulus of rupture in the evaluated treatments.

\begin{tabular}{|c|c|c|c|c|c|}
\hline \multirow{2}{*}{ Tratamentos } & \multicolumn{3}{|c|}{ Partículas (\%) } & \multirow{2}{*}{$\frac{\text { MOE }}{\text { (MPa) }}$} & \multirow{2}{*}{$\begin{array}{c}\text { MOR } \\
(\mathrm{MPa}) \\
\end{array}$} \\
\hline & Erva-mate & Pinus & Eucalipto & & \\
\hline $\mathrm{T} 1$ & 0 & 100 & 0 & $2208,82(188,41)$ & $13,74(0,98)$ \\
\hline T3 & 50 & 50 & 0 & $2214,32(188,88)$ & $10,36(0,74)$ \\
\hline T5 & 100 & 0 & 0 & $1181,45(100,77)$ & $4,84(0,34)$ \\
\hline T6 & 0 & 0 & 100 & $2065,37(176,17)$ & $13,67(0,98)$ \\
\hline T8 & 50 & 0 & 50 & $1749,80(149,25)$ & $9,30(0,66)$ \\
\hline T10 & 33,33 & 33,33 & 33,33 & $2101,46(179,25)$ & $11,43(0,81)$ \\
\hline $\mathrm{T} 11$ & 0 & 50 & 50 & $2435,49(207,74)$ & $14,50(1,04)$ \\
\hline
\end{tabular}

Onde: $\mathrm{MOE}$ = Módulo de elasticidade; MOR = Módulo de ruptura, entre parênteses desvio padrão associado a cada média. 
Os módulos analisados em flexão estática, apresentaram modelos de misturas com comportamentos distintos. O MOE apresentou efeito de interação binária, ou seja, o modelo foi quadrático, já o MOR foi explicado por modelo linear. Os coeficientes lineares de MOE e MOR e os coeficientes quadráticos de MOE interagiram de maneira sinérgica, contribuindo positivamente para estas propriedades mecânicas. Além disso, como todos os coeficientes lineares foram significativos para as referidas propriedades mecânicas, mostra que as três espécies que compõe a mistura tem participação nos resultados encontrados. Já para MOE, além de haver contribuição individual de cada espécie, houve interações entre espécies. Hillig, Haselein e Iwakiri (2003), estudando modelagem de misturas em painéis com diferentes proporções de eucalipto, pinus e acácia negra, encontraram coeficientes lineares significativos e sinérgicos, entretanto, observou somente modelos lineares, tanto para MOR quanto para MOE.

A análise dos resultados permitiu verificar que o MOE diminuiu com o acréscimo da proporção de partículas de erva-mate, o mesmo ocorreu no trabalho dos autores Carvalho et al. (2015) utilizando resíduo de erva-mate decorrente da poda. No modelo simplificado, o coeficiente linear para o teor de erva-mate tem valor menor $(1189,72)$ que os coeficientes derivados para o pinus $(2221,22)$ e eucalipto $(2073,64)$. Isso pode ter ocorrido em função do baixo Coeficiente de Esbeltes das partículas de erva-mate, 4,63 para a camada interna do painel e 9,98 para a camada externa. De acordo com Vital et al. (1992), partículas longas e finas (esbeltas) produzem painéis com maior resistência à flexão estática.

Quanto aos coeficientes quadráticos, houve interação entre erva-mate e pinus, assim como pinus e eucalipto, entretanto, a mistura mais eficiente para MOE foi entre erva-mate e pinus, com um coeficiente de 1903,09, valor semelhante aos coeficientes individuais de pinus e eucalipto.

A Figura 4a apresenta o gráfico ternário de $\mathrm{MOE}$ em razão da mistura dos três componentes. O referido gráfico representa a área de maiores valores de $\mathrm{MOE}$, a região com maior tonalidade vermelha, conforme esta tonalidade diminui e migra para região amarela e verde, diminui-se a elasticidade do painel. Desta maneira à medida que se aumenta a proporção de pinus ou eucalipto e diminui a proporção de erva-mate obtêm-se maiores valores de MOE. Entretanto, é possível visualizar por meio das curvas de nível que a mistura de partículas de erva-mate e pinus pode ser utilizada na proporção de até 50\%.
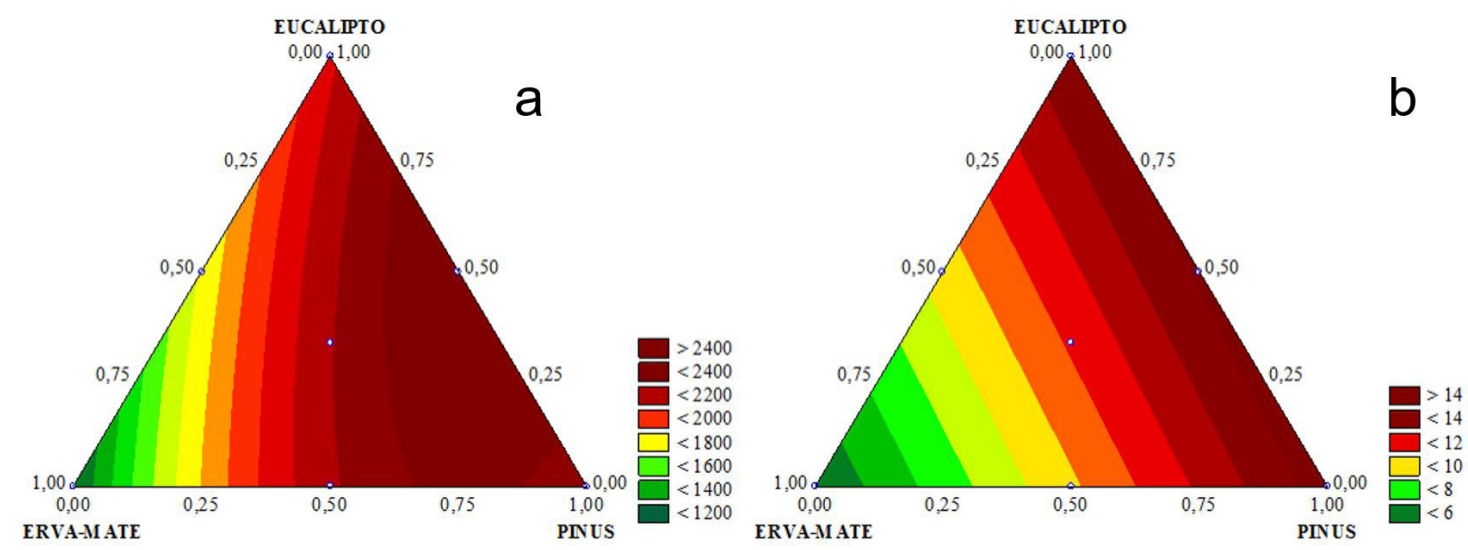

Figura 4 - a= gráfico ternário do módulo de elasticidade dos painéis, em função da mistura erva-mate, pinus e eucalipto, b= gráfico ternário do módulo de ruptura dos painéis, em função da mistura erva-mate, pinus e eucalipto.

Figure 4-a= Ternary graph of the modulus of elasticity of the panels, due to the mixture of yerba mate, pine and eucalyptus, $b=$ Ternary graph of the panel rupture modulus, due to the mixture of yerba mate, pine and eucalyptus.

Por meio do gráfico ternário da Figura $4 \mathrm{~b}$, pode-se observar que as propriedades de $\mathrm{MOE}$ e o MOR apresentaram comportamento semelhante, ou seja, assim como no MOE, o MOR apresentou os maiores valores para pinus $(14,52)$ e eucalipto $(13,83)$ e menor para erva-mate $(5,11)$. Na região verde, que representa os menores valores, se concentra mais em direção a erva-mate, à medida que se diminui a porcentagem da referida espécie e acrescenta-se pinus e/ou eucalipto, aumenta-se os valores de MOR. 
Talgatti et al. - Propriedades mecânicas do MDP produzido com partículas de

madeira de Ilex paraguariensis, Pinus elliottii e Eucalyptus grandis

A Tabela 4 apresenta os resultados médios de arrcancamento de parafuso na Face e de Topo dos painéis confeccionados com a mistura das diferentes espécies, erva-mate, pinus e eucalipto.

Tabela 4 - Modelos simplificados para o módulo de elasticidade e módulo de ruptura dos painéis, utilizando-se apenas os coeficientes significativos pelo teste " $t$ ".

Table 4 - Simplified models for the modulus of elasticity and modulus of rupture of the panels, using only the coefficients significant by the test " $t$ ".

\begin{tabular}{cccc}
\hline Modelo & $\mathbf{F}$ & P-valor & $\mathbf{R}^{2} \mathbf{C V}(\%)$ \\
\hline $\mathrm{MOE}=1189,72^{*} \mathrm{EM}+2221,22^{*} \mathrm{P}+2073,64^{*} \mathrm{E}$ & 10,58 & $0,0000^{* *}$ & $0,7038,53$ \\
$+1903,09^{*} \mathrm{EM} \mathrm{P}^{*}+1019,94^{*} \mathrm{P}^{*} \mathrm{E}$ & & & \\
$\mathrm{MOR}=5,11^{*} \mathrm{EM}+14,52^{*} \mathrm{P}+13,83^{*} \mathrm{E}$ & 137,13 & $0,0000^{* *}$ & $0,7857,18$ \\
\hline
\end{tabular}

Onde: *** = Significativo ao nível de $5 \%$ de probabilidade de erro; $\mathrm{F}=$ Valor de $\mathrm{F}$ calculado; $\mathrm{P}=$ Probabilidade; $\mathrm{R}^{2}$ = Coeficiente de determinação; $\mathrm{CV}=$ coeficiente de variação (\%); $\mathrm{EM}=$ Erva-mate; $\mathrm{P}$ = Pinus; $\mathrm{E}=$ Eucalipto; $\mathrm{MOE}=$ Módulo de elasticidade (MPa); $\mathrm{MOR}=$ Módulo de ruptura $(\mathrm{MPa})$.

Por meio da Tabela 4, observam-se valores de Arrcancamento de Parafuso. Assim como nas propriedades de flexão estática, ambas as propriedades mecânicas de AP tiveram valor mínimo para o tratamento 5, com 100\% de erva-mate. Sendo assim, os painéis fabricados com partículas madeireiras de erva-mate em grande proporção tiveram tendência a possuir valores inferiores para AP Face e AP Topo. Carvalho et al. (2015), analisando AP Face em painéis com partículas de erva-mate em combinação com pinus, observaram valores de 1452,40 a 1934,90 N, valores estes superiores aos encontrados no presente estudo, esta diferença pode ser atribuída a natureza das partículas utilizadas nos estudos, sendo dos autores supracitados resíduos de poda e do presente resíduo processado de ervateira.

Modes et al. (2012), avaliando painéis aglomerados de Peltophorum dubium com densidade nominal de $630 \mathrm{~kg} . \mathrm{m}^{-3}$, semelhante ao do presente trabalho, observaram o valor médio de 568,80 N para o Arrancamento do parafuso na Face. Trianoski et al. (2011) obtiveram os valores médios de 1482,96 e 1088,18 N para arrancamento do parafuso Face e Topo, respectivamente, em painéis puros de Acrocarpus fraxinifolius com densidade nominal de $750 \mathrm{~kg} . \mathrm{m}^{-3}$. Já Soratto et al. 2013, avaliando painéis MDP fabricados com eucalipto em associação com cavacos com casca, com densidade nominal de $650 \mathrm{~kg} . \mathrm{m}^{-3}$, observaram valores de Arrancamento do parafuso de 1532 a $2045 \mathrm{~N}$, sendo o menor valor para $0 \%$ de resíduo na confecção e o maior valor para cerca de $25 \%$ de adição, corroborando assim com o presente trabalho onde também foi verificado melhores resultados para esta propriedade utilizando-se resíduos.

Dessa forma, observa-se que o valor maior de densidade dos painéis pode resultar em melhores resultados mecânicos. Ainda, Trianoski et al. (2011) mencionaram que esta relação se deve a redução dos espaços vazios no painel, que promove um maior contato entre as partículas, gerando assim uma maior resistência mecânica.

A norma brasileira ABNT NBR 14810-2 (ABNT, 2013), estabelece valores mínimos de 1020 e 800 N, respectivamente para AP Face e AP Topo. Todos os tratamentos ficaram dentro do exigido ou pelo menos se assemelharam com a norma, exceto o tratamento 5, com 100\% erva-mate, que se apresentou inferior ao estabelecido pela norma brasileira para AP Topo. Portanto, assim como no MOE e MOR, embora a inserção de partículas erva-mate diminua as propriedades de AP Face e AP Topo, se utilizada à referida espécie em combinação com eucalipto e pinus, pode-se obter chapas com propriedades satisfatórias e atinge o mínimo exigido pela norma. Dentre os tratamentos com porcentagens de erva-mate, aquele com 33,33\% dos três componentes da mistura, mostrou maior eficiência, com valores superiores ao estabelecido pela ABNT para o arrancamento de parafuso.

Na Tabela 5, são apresentados os modelos simplificados, nos quais foram descartados os coeficientes não-significativos pelo teste " $\mathrm{t}$ " de student, a $5 \%$ de probabilidade de erro, para as propriedades de AP Face e AP Topo.

Entra Tabela 5.

Os arranchamentos de parafuso na face e no topo foram explicados por modelos lineares, com coeficientes sinérgicos. O mesmo foi constatado por Hillig Haselein e Iwakiri (2003), estudando modelagem de misturas em painéis com diferentes proporções de eucalipto, pinus e acácia negra. Os coeficientes lineares contribuíram de maneira positiva e semelhante para estas propriedades 
Tabela 5 -Valores médios de arrancamento de parafusos na face e topo, nos tratamentos avaliados. Table 5 - Average values of pulling of screws in the face and top, in the evaluated treatments.

\begin{tabular}{|c|c|c|c|c|c|}
\hline \multirow{2}{*}{ Tratamentos } & \multicolumn{3}{|c|}{ Partículas (\%) } & \multirow{2}{*}{$\begin{array}{c}\text { AP Face } \\
(\mathrm{N})\end{array}$} & \multirow{2}{*}{$\begin{array}{c}\text { AP Topo } \\
(\mathrm{N})\end{array}$} \\
\hline & Erva-mate & Pinus & Eucalipto & & \\
\hline T1 & 0 & 100 & 0 & $995,74(75,57)$ & $731,82(60,52)$ \\
\hline T3 & 50 & 50 & 0 & $993,09(75,37)$ & $700,69(57,94)$ \\
\hline T5 & 100 & 0 & 0 & $926,48(70,31)$ & $617,08(51,03)$ \\
\hline T6 & 0 & 0 & 100 & $1092,62(82,92)$ & $856,69(70,84)$ \\
\hline T8 & 50 & 0 & 50 & $999,54(75,86)$ & $722,50(59,75)$ \\
\hline T10 & 33,33 & 33,33 & 33,33 & $1034,19(78,49)$ & $806,52(66,69)$ \\
\hline $\mathrm{T} 11$ & 0 & 50 & 50 & $1047,27(79,48)$ & $935,64(77,37)$ \\
\hline
\end{tabular}

Onde: AP Face $=$ arrancamento de parafusos na face; AP Topo $=$ arrancamento de parafusos no topo, entre parênteses desvio padrão associado a cada média.

mecânicas, em especial para AP Face, em que o coeficiente de partículas de erva-mate $(946,66)$ ficou muito próximo ao de pinus $(1012,63)$ e eucalipto $(1088,22)$. Carvalho et al. $(2015)$, em seu estudo com painéis de erva-mate e pinus, encontraram valores próximos de AP Face entre estas duas espécies estudadas pelos autores supracitados.

As Figuras 5a e 5b exibem os gráficos ternários para AP Face e AP Topo, respectivamente, em que cores verdes representam valores mais baixos e amarelas e vermelhas, valores mais altos.
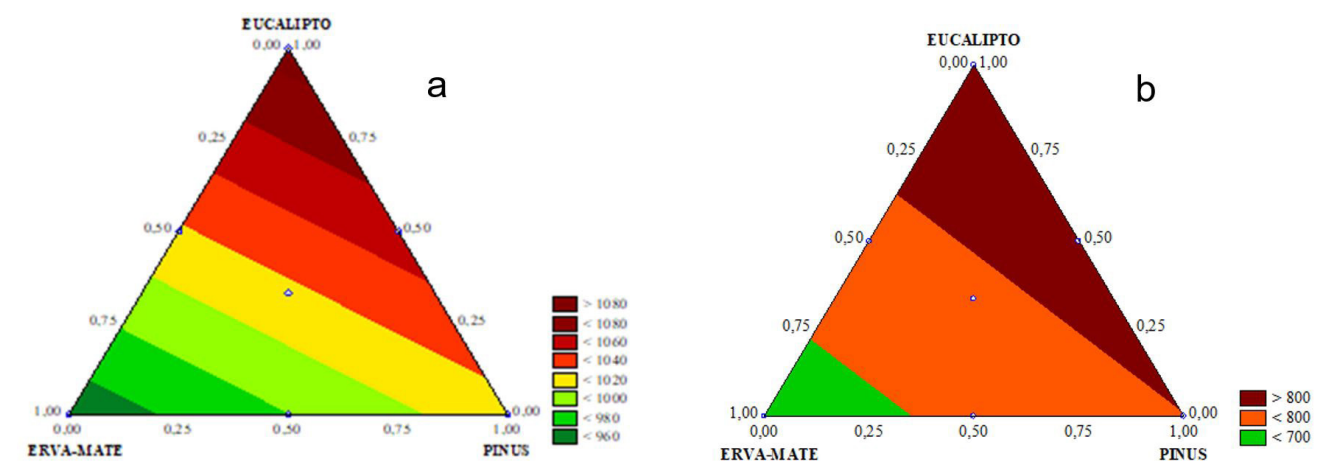

Figura 5 - a= gráfico ternário do arrancamento de parafusos na face dos painéis, em função da mistura erva-mate, pinus e eucalipto, $b=$ gráfico ternário do arrancamento de parafusos no topo dos painéis, em função da mistura erva-mate, pinus e eucalipto.

Figure 5 - Graph ternary of the pulling of screws in the face of the panels, due to the mixture of yerba mate, pine and eucalyptus, $b=$ Graph ternary of the pulling of screws at the top of the panels, due to the mixture of yerba mate, pine and eucalyptus.

Os valores de Tração Perpendicular-TP e Resistência superficial-RS apresentaram variação de 0,43 a 0,57 MPa e 0,59 a 1,11 MPa, respectivamente (Tabela 6). Desta vez, o valor mínimo foi para o tratamento $1 \mathrm{com} 100 \%$ de pinus, para ambas as propriedades mecânicas e para o tratamento 6 com $100 \%$ de eucalipto, para TP. As partículas de erva-mate colaboraram de maneira positiva para TP e RS, já que os tratamentos com presença da referida espécie possuem os valores mais altos, em especial para TP. Isto pode ter ocorrido por se tratar da adição de partículas menores e mais finas, partículas de resíduo de ervateira, o que incorre em maior compactação, diminuindo os espaços

Tabela 6 - Modelos simplificados para o arrancamento de parafusos na face e arrancamento de parafusos no topo dos painéis, utilizando-se apenas os coeficientes significativos pelo teste " $t$ ".

Table 6 - Simplified models for the pulling of screws on the face and pulling of screws at the top of the panels, using only the coefficients significant by the " $t$ " test.

\begin{tabular}{lcccc}
\hline \multicolumn{1}{c}{ Modelo } & $\mathbf{F}$ & P-valor & $\mathbf{R}^{2}$ & CV(\%) \\
\hline AP Face $=946,66^{*} \mathrm{EM}+1012,63^{*} \mathrm{P}+1088,22^{*} \mathrm{E}$ & 3,36 & $0,0398^{* *}$ & 0,151 & 7,59 \\
AP Topo $=646,67^{*} \mathrm{EM}+798,44^{*} \mathrm{P}+889,30^{*} \mathrm{E}$ & 9,25 & $0,0002^{* *}$ & 0,254 & 8,27
\end{tabular}

Onde: ** $=$ Significativo ao nível de $5 \%$ de probabilidade de erro; $\mathrm{F}=$ Valor de $\mathrm{F}$ calculado; $\mathrm{P}=$ Probabilidade; $\mathrm{R}^{2}=$ Coeficiente de determinação; $\mathrm{CV}=$ coeficiente de variação (\%); $\mathrm{EM}=$ Erva-mate; $\mathrm{P}=$ Pinus; $\mathrm{E}=$ Eucalipto; $\mathrm{AP}$ Face = arrancamento de parafusos na face $\mathrm{MPa}) ;$ AP Topo $=$ arrancamento de parafusos no topo $(\mathrm{MPa})$. 
vazios e a porosidade, resultando em uma melhor colagem. Corroborando Peixoto e Brito (2000), concluíram que painéis feitos com partículas menores apresentaram uma maior resistência à tração perpendicular quando comparados com aqueles que utilizam partículas maiores, isto é, as partículas menores são responsáveis por uma melhor uniformização do material e redução dos espaços internos.

Todavia, os autores a seguir utilizando espécies de cunho não convencional, encontraram valores superiores para a tração perpendicular: Iwakiri et al. (2010) 0,76 MPa (Schizolobium amazonicum); Trianoski et al. (2011), 1,12 MPa (Acrocarpus fraxinifolius); assim como Iwakiri et al. (2012b) que ao estudarem o resíduo de Ecclinusa guianensis na produção de painéis particulados, obtiveram o valor médio de 1,46 MPa. Já Soratto et al. (2013), na confecção de painéis MDP com resíduos de cavacos de eucalipto com casca e densidade nominal semelhante ao presente estudo, afeririam valores de resistência a Tração Perpendicular no intervalo de 0,50 a 0,97, resultados que corroboram com presente estudo que se encontram em média dentro do mesmo intervalo. Ainda, os autores supracitados mencionam que a adição de resíduo diminuiu esta propriedade em seus painéis, o oposto foi verificado no presente estudo com a adição de resíduo de erva-mate.

Saldanha (2004) citou que esta variação de resistência para TP, pode ocorrer em função da planicidade das partículas, bem como o índice de esbeltez. Tais características favorecem o processo de aplicação da resina, a formação do colchão e área de contato entre as partículas, ou seja, quanto maiores as razões de planicidade e esbeltez, melhores são as condições de distribuição da resina, o que pode interferir na resistência mecânica dos painéis.

De forma análoga, o baixo desempenho das partículas de eucalipto encontrado nos painéis desta pesquisa para TP, foi observado por Melo e Del Menezzi (2010). Os referidos autores relacionaram o baixo desempenho de painéis de Eucalyptus grandis ao gradiente vertical de densidade. Segundo os mesmos autores, esse gradiente pode ser influenciado pelo tipo de matéria-prima e fatores relacionados à prensagem, como tempo de prensagem, tempo de fechamento da prensa e pressão. Vital et al. (1992), ao estudar diferentes espécies de madeira utilizadas na manufatura de painéis aglomerados, verificaram que a resistência da TP foi influenciada pela densidade da camada interna do painel.

A norma brasileira ABNT NBR 14810-2 (ABNT, 2013), normatiza a TP e RS com valores mínimos de 0,35 MPa e $1 \mathrm{MPa}$, respectivamente. Todos os tratamentos ficaram dentro do exigido pela norma para o ensaio de TP, enquanto que para RS, três tratamentos (1, 3 e 5) ficaram abaixo do estabelecido pela ABNT. As partículas de erva-mate proporcionaram valores acima do normativo para TP e abaixo do esperado para RS. O tratamento 10, com 33,33\% dos três componentes das misturas, mostrou-se eficiente novamente, com valores superiores ao estabelecido pela norma brasileira.

Na Tabela 7, são apresentados os modelos simplificados, nos quais foram descartados os coeficientes não significativos pelo teste " $\mathrm{t}$ " de student, a 5\% de probabilidade de erro, para as propriedades de TP e RS.

Tabela 7 - Valores médios de tração perpendicular à superfície do painel e resistência superficial, nos tratamentos avaliados.

Table 7 - Average values of traction perpendicular to the surface of the panel and surface resistance, in the evaluated treatments.

\begin{tabular}{|c|c|c|c|c|c|}
\hline \multirow{2}{*}{ Tratamentos } & \multicolumn{3}{|c|}{ Partículas (\%) } & \multirow{2}{*}{$\begin{array}{c}\text { TP } \\
\text { (MPa) }\end{array}$} & \multirow{2}{*}{$\begin{array}{c}\text { RS } \\
\text { (MPa) }\end{array}$} \\
\hline & Erva-mate & Pinus & Eucalipto & & \\
\hline T1 & 0 & 100 & 0 & $0,43(0,04)$ & $0,59(0,04)$ \\
\hline T3 & 50 & 50 & 0 & $0,54(0,05)$ & $0,82(0,06)$ \\
\hline T5 & 100 & 0 & 0 & $0,57(0,05)$ & $0,90(0,07)$ \\
\hline T6 & 0 & 0 & 100 & $0,43(0,04)$ & $1,11(0,08)$ \\
\hline T8 & 50 & 0 & 50 & $0,48(0,04)$ & $0,98(0,07)$ \\
\hline T10 & 33,33 & 33,33 & 33,33 & $0,46(0,04)$ & $1,04(0,08)$ \\
\hline T11 & 0 & 50 & 50 & $0,51(0,04)$ & $1,11(0,08)$ \\
\hline
\end{tabular}

Onde: TP = tração perpendicular à superfície do painel; RS = resistência superficial, entre parênteses desvio padrão associado a cada média.

O ensaio mecânico de tração perpendicular, que mensura a resistência à tração na CI do painel, foi explicado por modelo linear, com coeficientes lineares e sinérgicos para as três espécies em análise, sendo assim, todos os ingredientes da mistura colaboraram nos resultados encontrados

A partir de uma breve análise do modelo para tração perpendicular, verifica-se que esta propriedade mecânica aumenta com o acréscimo da proporção de partículas de erva-mate (Tabela 8). No modelo simplificado, o coeficiente linear para o teor de erva-mate tem um valor maior $(0,5614)$ que os 
Tabela 8- Modelos simplificados para a tração perpendicular à superfície do painel e resistência superficial, utilizando-se apenas os coeficientes significativos pelo teste " $t$ ".

Table 8 - Simplified models for traction perpendicular to the surface of the panel and surface resistance, using only the coefficients significant by the " $t$ " test.

\begin{tabular}{ccccc}
\hline Modelo & $\mathbf{F}$ & $\mathbf{P}$ & $\mathbf{R}^{2}$ & CV (\%) \\
\hline $\mathrm{TP}=0,5614^{*} \mathrm{EM}+0,4689^{*} \mathrm{P}+0,4363^{*} \mathrm{E}$ & 6,51 & $0,0025^{* *}$ & 0,148 & 9,50 \\
$\mathrm{RS}=0,8969^{*} \mathrm{EM}+0,5918^{*} \mathrm{P}+1,1144^{*} \mathrm{E}+1,0261^{*} \mathrm{P}^{*} \mathrm{E}$ & 9,27 & $0,0000^{* *}$ & 0,577 & 8,09 \\
\hline
\end{tabular}

Onde: $* *$ = Significativo ao nível de $5 \%$ de probabilidade de erro; $\mathrm{F}=$ Valor de $\mathrm{F}$ calculado; $\mathrm{P}=$ Probabilidade; $\mathrm{R}^{2}$ = Coeficiente de determinação; $\mathrm{CV}=$ coeficiente de variação $(\%) ; \mathrm{EM}=$ Erva-mate; $\mathrm{P}=$ Pinus; $\mathrm{E}=$ Eucalipto; $\mathrm{TP}=$ tração perpendicular à superfície do painel (MPa); $\mathrm{RS}=$ resistência superficial $(\mathrm{MPa})$.

coeficientes derivados para o pinus $(0,4689)$ e eucalipto $(0,4363)$. Carvalho et al. (2015), estudando painéis puros e com misturas, entre erva-mate, resíduo de poda, e pinus, observaram o mesmo comportamento do presente estudo com resíduo de erva-mate de ervateira, pinus e eucalipto, ambos utilizando ureia-formaldeído.

O gráfico ternário para a tração perpendicular em razão da mistura dos três componentes está representado pela Figura 6a, em que pode ser observado os maiores valores de resistência mecânica para as partículas de erva-mate e os menores para eucalipto.
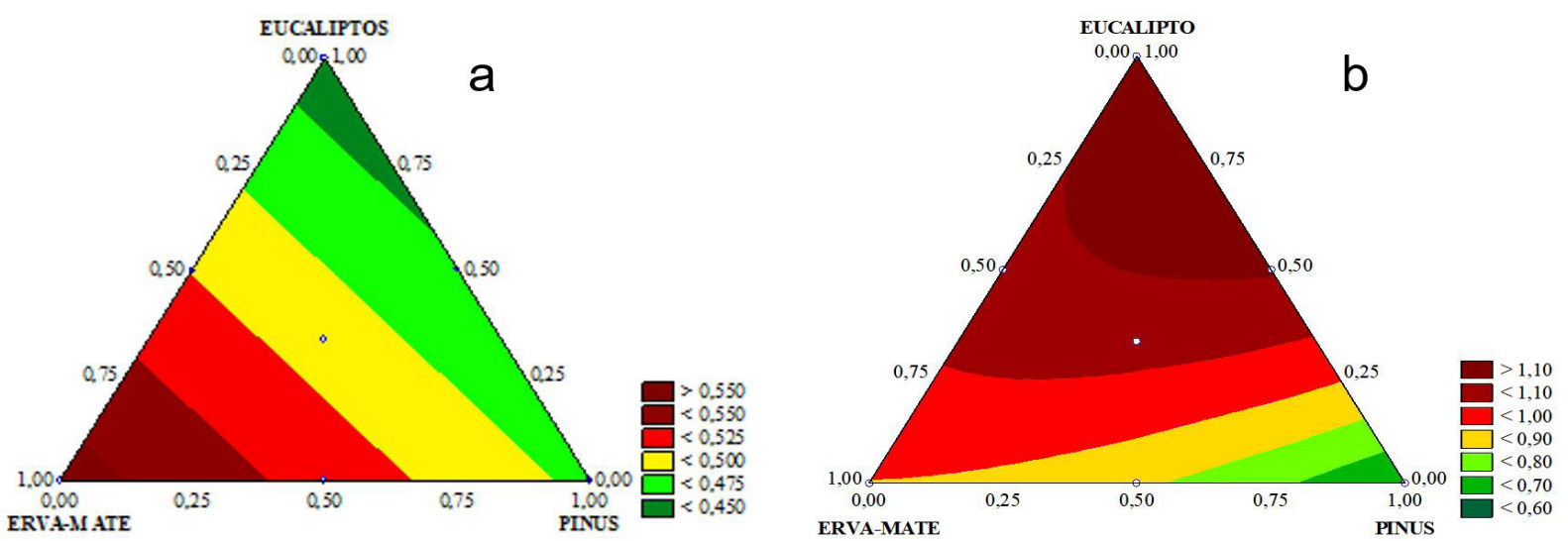

Figura 6 - a= gráfico ternário da tração perpendicular à superfície dos painéis, em função da mistura erva-mate, pinus e eucalipto, $b=$ gráfico ternário da resistência superficial dos painéis, em função da mistura erva-mate, pinus e eucalipto.

Figure $6-a=$ Ternary plot of the traction perpendicular to the surface of the panels, due to the mixture of yerba mate, pine and eucalyptus, $b=$ Termal graph of the surface resistance of the panels, due to the mixture of yerba mate, pine and eucalyptus.

Já o ensaio mecânico para RS, que mensura a resistência na CE do painel, foi explicado por modelo quadrático, apresentando efeito de interação binária. Assim como na TP, houve sinergia para os coeficientes lineares de RS, sendo assim, mais uma vez todos os ingredientes da mistura colaboraram nos resultados encontrados. A maior contribuição ficou por conta das partículas de eucalipto $(1,1144)$, seguida de erva-mate $(0,8969)$ e pinus $(0,5918)$, entretanto o coeficiente quadrático com interação binária entre pinus e eucalipto obteve elevada participação $(1,0261)$, muito semelhante à de eucalipto.

À medida que se aumenta a proporção de eucalipto obtêm-se maiores valores de RS, enquanto que, partículas de erva-mate obtêm-se valores intermediários e pinus valores mais baixos.

Sendo assim, este resíduo apresentou uma ótima performance quando combinado com pinus e eucalipto, apresentando valores satisfatórios e acima do exigido pela norma. Um exemplo disso, é o tratamento 10 , com $33,33 \%$ para os 3 componentes da mistura, erva-mate, pinus e eucalipto, que obteve valores superiores ao exigido pela ABNT, em todas as propriedades mecânicas.

\section{CONCLUSÕES}

A modelagem de misturas mostrou que o MOR de flexão estática, Arrancamento do Parafuso Face, Arrancamento do Parafuso Topo e Tração Perpendicular são determinados pelo modelo linear, ou seja, são influenciados apenas pelas características individuais de cada componente na mistura. 
Talgatti et al. - Propriedades mecânicas do MDP produzido com partículas de

madeira de Ilex paraguariensis, Pinus elliottii e Eucalyptus grandis

Por outro lado, as demais propriedades mecânicas são determinadas por modelo quadrático. No MOE de flexão estática, as partículas de erva-mate e pinus, e pinus e eucalipto, interagiram sinergicamente, ou seja, provocando um aumento dessa propriedade. Sendo a proporção mais indicada a mistura de $33,33 \%$ de cada espécie.

Já na Resistência Superficial houve interação sinérgica somente para pinus e eucalipto. Dessa forma, as propriedades que seguiram o modelo linear podem ser previstas por meio da soma das propriedades individuais de cada componente da mistura, enquanto as propriedades que seguiram o modelo quadrático devem levar em consideração a interação binária destes componentes.

De modo geral, o acréscimo do teor de partículas de erva-mate causou diminuição na maioria das propriedades mecânicas avaliadas no presente estudo. Comportamento inverso foi observado no ensaio de Tração Perpendicular. No entanto, somente o tratamento 5 com 100\% de erva-mate, obteve valores abaixo do exigido pela norma brasileira ABNT, na maior parte dos ensaios mecânicos.

Os painéis puros com resíduos madeireiros de erva-mate não apresentaram qualidades tecnológicas satisfatórias. Entretanto este resíduo em combinação com as espécies comumente utilizadas em painéis comerciais, pinus e eucalipto, pode ser utilizado em painéis MDP sem prejudicar a qualidade dos mesmos. Desta maneira, as partículas madeireiras de erva-mate apresentaram desempenho satisfatório em misturas com espécies tradicionais neste ramo de produção, apresentando valores satisfatórios e acima do exigido pela norma de comercialização, tanto para as propriedades físicas quanto para as mecânicas.

\section{REFERÊNCIAS BIBLIOGRÁFICAS}

ABIPA - ASSOCIAÇÃO BRASILEIRA DA INDÚSTRIA DE PAINÉIS AGLOMERADOS. Nossas unidades industriais. 2015. Disponível em: < http://www.abipa.org.br/industrias.php >. Acesso em: 12 fev. 2015.

ABNT - ASSOCIAÇÃO BRASILEIRA DE NORMAS TÉCNICAS. NBR 14810: painéis de madeira de média densidade. Rio de Janeiro, p. 69, 2013. 69 p.

BALDIN, T.; SILVEIRA, A. G.; VIDRANO, B. R. A.; CANCIAN, L. C.; SPATT, L. L.; HASELEIN, C. R. Qualidade de painéis aglomerados produzidos com diferentes proporções de madeira e capim-annoni. Revista Brasileira de Ciências Agrárias, Recife, v. 11, n. 3, p. 230-237, 2016.

BIAZUS, A.; HORA, A. B.; LEITE, B. G. P. Panorama de mercado: painéis de madeira. BNDES Setorial, Rio de Janeiro, n. 32, p.49-90, 2010.

BORSATO, D.; DALL'ANTONIA, L. H.; GUEDES, C. L. B.; MAIA, E. C. R.; FREITAS, H. R. DE; MOREIRA, I.; SPACINO, K. R. Aplicação do delineamento simplex-centroide no estudo da cinética da oxidação de biodiesel b100 em mistura com antioxidantes sintéticos. Química Nova, São Paulo, v. 33, n. 8, p.1726-1731, 2010.

CARVALHO, A. G.; ANDRADE, B. G.; CABRAL, C. P. T.; VITAL, B. R. Efeito da adição de resíduos de poda da erva-mate em painéis aglomerados. Revista Árvore, Viçosa, v. 39, n. 1, p. 209-214, 2015.

GUIMARÃES JÚNIOR. R.; ARAÚJO, B. L. M.; LOPES, O. P.; MENDES, R. F.; MENDES, L. M. Produção de painéis aglomerados da madeira de desrama de Acacia mangium. Pesquisa Florestal Brasileira, Colombo, v. 33, n. 76, p. 387-391, 2013.

HILLIG, E.; HASELEIN, C. R.; IWAKIRI, S. Modelagem de mistura de três espécies de madeiras na fabricação de chapas estruturais. Floresta, Curitiba, v. 33, n. 3, p. 311 - 320, 2003.

HILLIG, E.; CARVALHO, C. A.; SCHNEIDER, V. E.; POCAI, K. Modelagem de misturas na fabricação de compósitos polímero-fibra, utilizando polietileno e serragem de Pinus sp. Ciência Florestal, Santa Maria, v. 16, n. 3, p. 343-351, 2006.

IBÁ - INDÚSTRIA BRASILEIRA DE ÁRVORES. Relatório anual 2015: ano base 2014. Brasília: IBÁ, 2015.

IWAKIRI, S.; TRIANOSKI, R.; CHIES, D.; TAVARES, E. L.; FRANÇA, M. C.; LAU, P. C.; IWAKIRI, V. T. Use of residues of forestry exploration of Pinus taeda for particleboard manufacture. Revista Árvore, Viçosa, v. 41, n. 3, p.1-8, 2017. 
IWAKIRI, S.; MATOS, J. L. M.; TRIANOSKI, R.; PRATA, J. G. Produção de painéis aglomerados homogêneos e multicamadas de Melia azedarach (cinamomo) e Pinus taeda com diferentes teores de resina. Cerne, Lavras, v. 18, n. 3, p. 465-470, 2012a.

IWAKIRI, S.; VIANEZ, B. F.; WEBER, C.; TRIANOSKI, R.; ALMEIDA, V. C. Avaliação das propriedades de painéis aglomerados produzidos com resíduo de serrarias de nove espécies de madeiras tropicais da Amazônia. Acta Amazônica, Manaus, v. 42, n. 1, p. 59-64, mar. 2012b.

IWAKIRI, S.; ZELLER, F.; PINTO, J. A.; RAMIREZ, M. G. L.; SOUZA, M. M.; SEIXAS, R. Avaliação do potencial de utilização da madeira de Schizolobium amazonicum "Paricá" e Cecropia hololeuca "Embaúba" para produção de painéis aglomerados. Acta Amazônica, Manaus, v. 40, n. 2, p. 303-308, 2010.

MELO, R. R.; DEL MENEZZI, C. H. S. Influência da massa específica nas propriedades físico-mecânicas de painéis aglomerados. Silva Lusitana, Lisboa, v. 18, p. 59-73, 2010.

MENDES, R. F.; MENDES, L. M.; GUIMARÃES JUNIOR, J. B.; SANTOS, R. C.; BUFALINO, L. The adhesive effect on the properties of particleboards made from sugar cane generated in the distiller. Revista Brasileira de Ciências Agrárias, Recife, v. 32, n. 2, p. 209-218, 2009.

MODES, K. S.; VIVIAN, M. A.; LILGE, D. S.; MELO, R. R.; SANTINI, E. J.; HASELEIN, C. R. Utilização da madeira de canafístula (Peltophorum dubium (Spreng.) Taub.) na confecção de chapas de madeira aglomeradas. Ciência Florestal, Santa Maria, v. 22, n. 1, p. 147-159, 2012.

MURUGANANDAM, L.; RANJITHA, J.; HARSHAVARDHAN, A. A review report on physical and mechanical properties of particle boards from organic waste. International Journal of ChemTech Reserarch, Mumbai, v. 9, n. 1, 2016.

PEIXOTO, G. L; BRITO, E. O. Avaliação da granulometria de partículas de Pinus taeda combinadas com adesivos comerciais para a fabricação de aglomerados. Floresta e Ambiente, Seropédica, v. 7, n. 1, p. 60-67, 2000.

SALDANHA, L. K. Alternativas tecnológicas para produção de chapas de partículas orientadas "OSB". 2004. 83 p. Dissertação (Mestrado em Ciências Florestais) - Universidade Federal do Paraná, Curitiba, 2004.

SANCHES, F. L.; HILLIG, E.; IWAKIRI, S.; NAPOL, L. M. Resistência de painéis aglomerados produzidos com mistura de madeira de espécies florestais tradicionais e não tradicionais. Ciência Florestal, Santa Maria, v. 26, n. 2, p. 559-569, 2016.

SILVA, D. W.; FARRAPO, C. L.; RIBEIRO, D. P.; MENDES, R. F.; MENDES, L. M.; SCOLFORO, J. R. S. MDP com partículas de eucalipto e palha de milho. Scientia Forestalis, Piracicaba, v. 43, n. 108, p. 853-862, 2015.

SORATTO, D. N.; CUNHA, A. B.; VITAL, B. R.; CARNEIRO, A. C. O.; COSTA, F. R. Efeitos da adiçao de cavaco com casca na qualidade e painéis MDP produzidos com Eucalyptus sp. Ciência da Madeira, Pelotas, v. 4, n. 1, p. 46-59, 2013.

TALGATTI, M. BALDIN, T.; SILVEIRA, A. G. SANTINI, E. J.; VIDRANO, B. R. A. Compósito madeira-plástico a partir de resíduos de três espécies florestais. Pesquisa Florestal Brasileira, Colombo, v. 37, n. 91, p. 277283, 2017.

TORRELL, R.; HILLIG, E.; CORRADI, G.; IWAKIRI, S. Influência da adição de serragem nas propriedades tecnológicas de painéis de madeira aglomerada de Pinus taeda. Ambiência, Guarapuava, v.9 n.1 p. 57 - $72,2013$.

TRIANOSKI, R.; IWAKIRI, S.; MATOS, J. L. M.; PRATA, J. G. Viabilidade da utilização de Acrocarpus fraxinifolius em diferentes proporções com Pinus spp. para produção de painéis aglomerados. Scientia Forestalis, Piracicaba, v. 39, n. 91, p. 343-350, 2011.

VITAL, B. R.; HASELEIN, C. R.; DELLA LUCIA, R. M. Efeito da geometria das partículas nas propriedades dos painéis de madeira aglomerada de Eucalyptus grandis (Hill ex-Maiden). Revista Árvore, Viçosa, v. 16, n. 1, p. 88-96, 1992.

Recebido em: 02/11/2017

Aceito em: 24/09/2018

Sci. For., Piracicaba, v. 47, n. 122, p. 273-285, jun. 2019

DOI: doi.org/10.18671/scifor.v47n122.10 